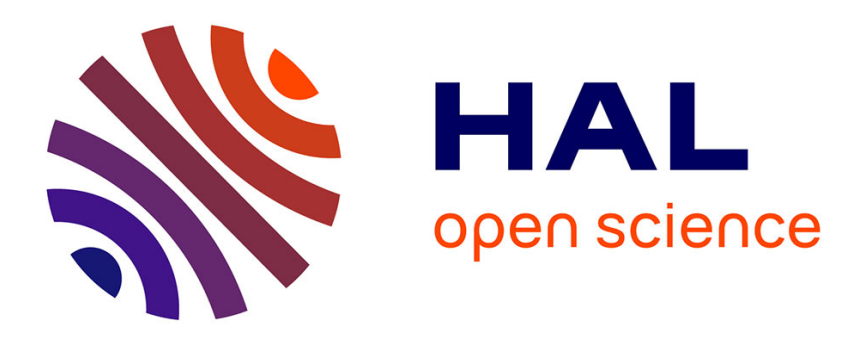

\title{
Path integrals and time-dependent mean-field theories
}

\author{
Jean-Paul Blaizot, Henri Orland
}

\section{To cite this version:}

Jean-Paul Blaizot, Henri Orland. Path integrals and time-dependent mean-field theories. Journal de Physique Lettres, 1980, 41 (3), pp.53-56. 10.1051/jphyslet:0198000410305300 . jpa-00231720

\section{HAL Id: jpa-00231720 https://hal.science/jpa-00231720}

Submitted on 1 Jan 1980

HAL is a multi-disciplinary open access archive for the deposit and dissemination of scientific research documents, whether they are published or not. The documents may come from teaching and research institutions in France or abroad, or from public or private research centers.
L'archive ouverte pluridisciplinaire HAL, est destinée au dépôt et à la diffusion de documents scientifiques de niveau recherche, publiés ou non, émanant des établissements d'enseignement et de recherche français ou étrangers, des laboratoires publics ou privés. 


\title{
Path integrals and time-dependent mean-field theories
}

\author{
J.-P. Blaizot and H. Orland \\ Service de Physique Théorique, C.E.N. de Saclay, B.P.nº 2,91190 Gif sur Yvette, France
}

(Reçu le 19 décembre 1979, accepté le 20 décembre 1979)

\begin{abstract}
Résumé. - On présente une formulation du problème à $N$-corps à l'aide d'une intégrale fonctionnelle définie sur un ensemble continu et surcomplet d'état dans l'espace de Hilbert. Le calcul de cette intégrale par la méthode du col conduit à des équations de mouvement classiques qui peuvent être quantifiées suivant une procédure bien définie. Les équations de Hartree-Fock dépendant du temps sont un exemple de telles équations classiques. Elles sont obtenues en intégrant sur un ensemble surcomplet de déterminants de Slater.
\end{abstract}

\begin{abstract}
We present a path integral formulation of the many-body problem, using a continuous and overcomplete set of vectors in the Hilbert space. The saddle-point approximation for the calculation of the functional integral leads to classical equation of motion which can be quantized in a well prescribed way. The time dependent Hartree-Fock equations are an example of such classical equations. They are obtained by integrating over an overcomplete set of Slater determinant.
\end{abstract}

Path integrals have shown to be one of the most powerful tools in quantum field theory and statistical mechanics. They allow operations on the perturbation expansion which are difficult to obtain in terms of the Feynmann graphs and lead to new approximation schemes. The application of such techniques to the nuclear many-body problem has recently been considered, in particular in connection with the quantization of the time dependent Hartree-Fock equations [1, 2]. However, all the formulations presented up to now suffer from one drawback : the exchange term of the two-body interaction does not appear on the same footing as the direct one. For applications to nuclear physics, this is a serious limitation. Some attempts have been made to remedy this defect, at least in lowest order. However, the solutions which have been proposed [2] suffer from a lack of generality or involve a rather artificial decomposition of the two-body interaction, which is actually not necessary as we shall show. In fact these ad-hoc treatments hide the real nature of the difficulty.

In this note, we present a new approach to the problem. We derive a path integral for the many-body problem using continuous and overcomplete sets of states in the Hilbert space. We discuss in particular the time dependent Hartree-Fock approximation and we show that antisymmetrization is correctly preserved at all levels of approximation. Moreover, our formulation gives a clear physical insight into the classical nature of the solutions of the time dependent Hartree-Fock equations and it provides a definite prescription to extract from them information about quantum processes.

We now proceed to the presentation of the most significant results we have obtained so far. The details of the derivation and a more complete discussion will be given in a forthcoming publication.

Let $\{|\psi(z)\rangle\}$ be an overcomplete set of vectors in the Hilbert space $\mathscr{H}$, depending upon a family of parameters, generally complex, which we denote collectively by $z$. We mean that there exists a measure $\mathrm{d} \mu(z)$ on the space where $z$ is defined, such that :

$$
\int \mathrm{d} \mu(z)|\psi(z)\rangle\langle\psi(z)|=\mathbb{1}
$$

where 1 denotes the unity operator in $\mathcal{H}$. In the following we note $|z\rangle$ the states $|\psi(z)\rangle$. We use the set $\{|z\rangle\}$ to write a functional representation for the matrix element of the evolution operator $\mathrm{e}^{-i \boldsymbol{H} t}$ between some initial state $\left|z_{\mathrm{i}}\right\rangle$ and some final state $\left|z_{\mathbf{f}}\right\rangle$ :

$$
U\left(z_{\mathrm{f}}, t_{\mathrm{f}} ; z_{\mathrm{i}} t_{\mathrm{i}}\right)=\left\langle z_{\mathrm{f}}\left|\mathrm{e}^{-i H\left(t_{\mathrm{f}}-t_{\mathrm{i}}\right)}\right| z_{\mathrm{i}}\right\rangle .
$$

First, we factorize $\mathrm{e}^{-i H\left(t_{\mathrm{f}}-t_{\mathrm{i}}\right)}$ into $N+1$ terms $\mathrm{e}^{-i \varepsilon H}$, where $\varepsilon=\frac{t_{\mathrm{f}}-t_{\mathrm{i}}}{N+1}$. Then (1) is inserted between each of the operators, and we get : 


$$
U\left(z_{\mathrm{f}}, t_{\mathrm{f}} ; z_{\mathrm{i}}, t_{\mathrm{i}}\right)=\int \prod_{k=1}^{N} \mathrm{~d} \mu\left(z_{k}\right)\left\langle z_{\mathrm{f}}\left|\mathrm{e}^{-i \varepsilon H}\right| z_{N}\right\rangle \cdots\left\langle z_{k+1}\left|\mathrm{e}^{-i \varepsilon H}\right| z_{k}\right\rangle \cdots\left\langle z_{1}\left|\mathrm{e}^{-i \varepsilon H}\right| z_{\mathrm{i}}\right\rangle .
$$

In the limit where $N \rightarrow \infty, \varepsilon \rightarrow 0$ and :

$$
\left\langle z_{k+1}\left|\mathrm{e}^{-i \varepsilon H}\right| z_{k}\right\rangle \simeq\left\langle z_{k+1} \mid z_{k}\right\rangle \exp \left(-i \varepsilon \frac{\left\langle z_{k+1}|H| z_{k}\right\rangle}{\left\langle z_{k+1} \mid z_{k}\right\rangle}\right)+0\left(\varepsilon^{2}\right) .
$$

Thus we arrive at the following result :

$$
U\left(z_{\mathrm{f}}, t_{\mathrm{f}} ; z_{\mathrm{i}}, t_{\mathrm{j}}\right)=\lim _{N \rightarrow \infty} \int \prod_{k=1}^{N} \mathrm{~d} \mu\left(z_{k}\right) \prod_{k=0}^{N}\left\langle z_{k+1} \mid z_{k}\right\rangle \exp \left(-i \varepsilon \sum_{k=0}^{N} \frac{\left\langle z_{k+1}|H| z_{k}\right\rangle}{\left\langle z_{k+1} \mid z_{k}\right\rangle}\right)
$$

with the convention :

$$
\left\langle z_{N+1}\left|\equiv\left\langle z_{\mathbf{f}}|\quad| z_{0}\right\rangle \equiv\right| z_{\mathrm{i}}\right\rangle
$$

In order to proceed further, we consider the case where $\{|z\rangle\}$ is a set of states describing a system of $N$ identical Fermions (Slater determinants). Let $\left|\varphi_{0}\right\rangle$ be such a state. By convention, we call hole states the single particle states occupied in $\left|\varphi_{0}\right\rangle$, and particle states the other. Thus $\left|\varphi_{0}\right\rangle$ can be written :

$$
\left|\varphi_{0}\right\rangle=\prod_{h=1}^{N} a_{h}^{+}|0\rangle
$$

The states :

$$
|z\rangle=\exp \left(\sum_{p h} z_{p h} a_{p}^{+} a_{h}\right)\left|\varphi_{0}\right\rangle
$$

form an overcomplete set of Slater determinants [3]. A particular choice of a measure yields the following closure relation :

$$
\int \prod_{p h} \frac{\mathrm{d} z_{p h}^{*} \mathrm{~d} z_{p h}}{2 \pi i} \exp \left(-\sum_{p h} z_{p h}^{*} z_{p h}\right)|z\rangle\langle z|=1
$$

where $z_{p h}^{*}$ and $z_{p h}$ have to be considered as two independent integration variables :

$$
\frac{\mathrm{d} z^{*} \mathrm{~d} z}{2 \pi i}=\frac{\mathrm{d} \operatorname{Re}(z) \mathrm{d} \operatorname{Im}(z)}{\pi} .
$$

The Hamiltonian of the systems which we consider is the sum of one-body and two-body operators :

$$
H=\sum_{\alpha \beta} H_{\alpha \beta}^{0} a_{\alpha}^{+} a_{\beta}+\frac{1}{4} \sum_{\alpha \beta \gamma \delta}\langle\alpha \beta|V| \gamma \delta\rangle a_{\alpha}^{+} a_{\beta}^{+} a_{\delta} a_{\gamma} .
$$

The quantity $\langle\alpha \beta|V| \gamma \delta\rangle$ denotes the antisymmetrized matrix element of the two-body interaction $V$. The matrix element of $H$ between two states $\left\langle z_{k+1}\right|$ and $\left|z_{k}\right\rangle$ is easily evaluated in terms of the one-body density matrix $\rho$ defined by :

$$
\rho_{\alpha \beta}(k+1)=\frac{\left\langle z_{k+1}\left|a_{\beta}^{+} a_{\alpha}\right| z_{k}\right\rangle}{\left\langle z_{k+1} \mid z_{k}\right\rangle} .
$$

One has indeed :

$$
\frac{\left\langle z_{k+1}|H| z_{k}\right\rangle}{\left\langle z_{k+1} \mid z_{k}\right\rangle}=\sum_{\alpha \beta} H_{\alpha \beta}^{0} \rho_{\beta \alpha}(k+1)+\frac{1}{2} \sum_{\alpha \beta \gamma \delta}\langle\alpha \beta|V| \gamma \delta\rangle \rho_{\gamma \alpha}(k+1) \rho_{\delta \beta}(k+1)=H^{0} . \rho+\frac{1}{2} \rho . V . \rho
$$

where we use an obvious matrix notation. Putting (12) into (5) and using the following identity :

$$
\frac{\int \mathrm{d} W \exp \left(+i \frac{\varepsilon}{2} W \cdot V^{-1} \cdot W-i \varepsilon W \cdot \rho\right)}{\int \mathrm{d} W \exp \left(i \frac{\varepsilon}{2} W \cdot V^{-1} \cdot W\right)}=\exp \left(-i \frac{\varepsilon}{2} \rho \cdot V \cdot \rho\right)
$$

with $W$ a real quantity, one obtains the following functional integral for $U$ :

$$
\begin{aligned}
U\left(z_{\mathrm{f}}, t_{\mathrm{f}} ; z_{\mathrm{i}}, t_{\mathrm{i}}\right)=\mathcal{N} \lim _{N \rightarrow \infty} \int_{k=1}^{N} \mathrm{~d} W_{k} \exp \left(i \frac{\varepsilon}{2} \sum_{k=0}^{N} W_{k+1} \cdot V^{-1} \cdot W_{k+1}\right) \tilde{U}_{0}\left(z_{\mathrm{f}}, t_{\mathrm{f}} ; z_{\mathrm{i}}, t_{\mathrm{i}}\right) \\
\begin{array}{r}
\tilde{U}_{0}\left(z_{\mathrm{f}}, t_{\mathrm{f}} ; z_{\mathrm{i}}, t_{\mathrm{i}}\right)=\lim _{N \rightarrow \infty} \int \prod_{k=1}^{N} \frac{\mathrm{d} z_{k}^{*} \mathrm{~d} z_{k}}{2 \pi i} \exp \left(-\operatorname{Tr} z_{k}^{+} \cdot z_{k}\right) \prod_{k=0}^{N}\left\langle z_{k+1} \mid z_{k}\right\rangle \times \\
\times \exp \left[-i \varepsilon \sum_{k=0}^{N} H^{0} \cdot \rho(k+1)+W_{k+1} \cdot \rho(k+1)\right] .
\end{array}
\end{aligned}
$$


In $(14 a)$ the quantity $\mathcal{N}$ is a normalization constant. $\tilde{U}_{0}$ is very similar to the evolution operator of a system of non-interacting particles in an external field $W(t)$. Let $U_{0}$ be this operator :

$$
\begin{aligned}
& U_{0}\left(z_{\mathrm{f}}, t_{\mathrm{f}} ; z_{\mathrm{i}}, t_{\mathrm{i}}\right)= \\
& \quad=\left\langle z_{\mathrm{f}}\left|\mathrm{T} \exp -i \int_{t_{\mathrm{i}}}^{t_{\mathrm{f}}}\left[H^{0}+W(t)\right] \mathrm{d} t\right| z_{\mathrm{i}}\right\rangle .
\end{aligned}
$$

Using the same techniques as before, one can obtain a functional integral representation for $U_{0}\left(z_{\mathrm{f}}, t_{\mathrm{f}} ; z_{\mathrm{i}}, t_{\mathrm{i}}\right)$ which is precisely the same as that for $\tilde{U}_{0}$ given by eq. $(14 b)$. $U_{0}$ and $\tilde{U}_{0}$ cannot be identified however, for the following reason : they do not operate in the same way in the integral over the auxiliary field $W$ (eq. (14a)). The origin of the difficulty lies in the fact that the contributions to the integral (14a) come from terms which are of order $\varepsilon^{2}$ in $\tilde{U}_{0}$, and which are typically of the form :

$$
-\frac{\varepsilon^{2}}{2} W_{\alpha \beta} \rho_{\beta \alpha} W_{\gamma \delta} \rho_{\delta \gamma}
$$

Had one used $U_{0}$ in place of $\tilde{U}_{0}$, one would have obtained two terms corresponding to the two possible contractions :

$$
\begin{aligned}
-\frac{\varepsilon^{2}}{2} W_{\alpha \beta} W_{\gamma \delta}\left(\left\langle a_{\alpha}^{+} a_{\beta}\right\rangle\right. & \left\langle a_{\gamma}^{+} a_{\delta}\right\rangle+ \\
& \left.+\left\langle a_{\alpha}^{+} a_{\delta}\right\rangle\left\langle a_{\beta} a_{\gamma}^{+}\right\rangle\right)
\end{aligned}
$$

where $\left\langle a_{\alpha}^{+} a_{\beta}\right\rangle$ stands for $\frac{\left\langle z_{k+1}\left|a_{\alpha}^{+} a_{\beta}\right| z_{k}\right\rangle}{\left\langle z_{k+1} \mid z_{k}\right\rangle}$. Therefore the use of $U_{0}$ in place of $\tilde{U}_{0}$ in the integral (14a) leads to overcounting. This is an important point which has to be kept in mind if one wishes to recover from $(14 a)$ the usual perturbation expansion in powers of the two-body interaction $V$. It also prevents us of giving for $\tilde{U}_{0}$ a simple operator representation.

However, all these complications disappear when one calculates the integral $(14 a)$ in the saddle point approximation. Then one has to look for fields $W$ which make stationary the following effective action :

$S[W]=\frac{i}{2} \int_{t_{\mathrm{i}}}^{t_{\mathrm{f}}} W(t) \cdot V^{-1} \cdot W(t)+\log \tilde{U}_{0}\left(z_{\mathrm{f}}, t_{\mathrm{f}} ; z_{\mathrm{i}}, t_{\mathrm{i}}\right)$.

Such fields $W$ are solution of the equation :

$$
\frac{\delta S}{\delta W}=i V^{-1} W+\frac{1}{\tilde{U}_{0}} \frac{\delta \tilde{U}_{0}}{\delta W}=0 .
$$

It is easily shown that :

$$
\frac{1}{\tilde{U}_{0}} \frac{\delta \tilde{U}_{0}}{\delta W}=\frac{1}{U_{0}} \frac{\delta U_{0}}{\delta W}
$$

Furthermore, $\frac{1}{U_{0}} \frac{\delta U_{0}}{\delta W(t)}$ is proportional to the density matrix at time $t$ :

$$
\begin{aligned}
\frac{1}{U_{0}} \frac{\delta U_{0}}{\delta W_{\alpha \beta}(t)} & =-i \frac{\left\langle z_{\mathrm{f}}\left|U_{0}\left(t_{\mathrm{f}}, t\right) a_{\alpha}^{+} a_{\beta} U_{0}\left(t, t_{\mathrm{i}}\right)\right| z_{\mathrm{i}}\right\rangle}{\left\langle z_{\mathrm{f}}\left|U_{0}\left(t_{\mathrm{f}}, t_{\mathrm{i}}\right)\right| z_{\mathrm{i}}\right\rangle} \\
& =-i \rho(t) .
\end{aligned}
$$

From the eqs. (21a) and (21b) one easily derives the equation of motion for $\rho$ :

$$
i \dot{\rho}=\left[H_{0}+W \cdot \rho\right]
$$

and from (19), (20) and (21) one gets :

$$
W=V \cdot \rho \text {. }
$$

The eqs. (22) and (23) are therefore the time-dependent Hartree-Fock equations, with the exchange term correctly included in the mean field $W$.

A few comments are now in order. First of all, the density matrix given by eq. (21) depends upon the initial and final states. The same is true of the mean field $W$. This is a very important feature which contrasts with the usual time-dependent HartreeFock approximation. The latter can be recovered by assuming that at any time $t$ the state of the system is :

$$
|z(t)\rangle=U_{0}\left(t, t_{\mathrm{i}}\right)\left|z_{\mathrm{i}}\right\rangle \text {. }
$$

In particular the final state $\left|z_{\mathrm{f}}\right\rangle=U_{0}\left(t_{\mathrm{f}}, t_{\mathrm{i}}\right)\left|z_{\mathrm{i}}\right\rangle$; in general $\left|z_{\mathrm{f}}\right\rangle$ is not a stationary state which makes the interpretation of standard TDHF calculations quite difficult. The eqs. (22) and (23), with the correct boundary conditions, offer an interesting tool for the direct calculation of transition amplitudes between given initial and final states. Our second comment concerns the application of the saddle point approximation for the calculation of the functional integral $(14 a)$. To the extent that the functional integral $(14 a)$ is completely equivalent to the formal perturbation expansion in powers of $V$, one may consider the saddle point approximation, and its successive corrections, as a formal device to generate classical equation of motion and a definite prescription to quantize them. This is the point of view which we adopt. Finally we would like to mention that we have failed in all our tentatives to derive the TDHF equations using identities like (13) with density matrices replaced by Fermion pair operators. One typically ends up with a mean field theory which do not take into account the exchange contribution of the two-body interaction, this one appearing as a correction to the saddle point approximation. This difficulty is connected with the fact that there is no simple operator which can be associated with $\tilde{U}_{0}$ (eq. (14b)).

The authors would like to thank R. Balian for many usefull discussions. 


\section{References}

[1] Kleinert, H., Phys. Lett. 69B (1977) 9. Reinhardt, H., Nucl. Phys. A 298 (1978) 77; Nucl. Phys. A 306 (1978) 38.

[2] Levit, S., Negele, J. W. and Paltiel, Z., MIT Preprint (may 1979).

[3] Thouless, D. J., Nucl. Phys. 21 (1960) 225. 